\title{
Nanowell Array based Sensor and Its Packaging
}

\author{
JuKyung Lee ${ }^{1}$, Tsuda Akira ${ }^{2}$, Myung Yung Jeong ${ }^{3}$ and Hea Yeon Lee ${ }^{1, \dagger}$ \\ ${ }^{1}$ Department of Mechanical and Industrial Engineering, College of Engineering, Northeastern University, Boston, MA02115, USA \\ ${ }^{2}$ Department of Environmental Health, Harvard School of Public Health, Boston, MA 02115, USA \\ ${ }^{3}$ Department of Congo-Mechatronics Engineering, Pusan National University, Busan 609-735, Korea
}

(Received September 5, 2014: Corrected September 18, 2014: Accepted September 19, 2014)

\begin{abstract}
This article reviews the recent progress in nanowell array biosensors that use the label-free detection protocol, and are detected in their natural forms. These nanowell array biosensors are fabricated by nanofabrication technologies that should be useful for developing highly sensitive and selective also reproducible biosensors. Moreover, electrochemical method was selected as analysis method that has high sensitivity compared with other analysis. Finally, highly sensitive nanobiosensor was achieved by combining nanofabrication technologies and classical electrochemical method. Many examples are mentioned about the sensing performance of nanowell array biosensors will be evaluated in terms of sensitivity and detection limit compared with other micro-sized electrode without nanowell array.
\end{abstract}

Keywords: Nanowell Arrays (NWA), Nanobiosensor, Electrochemical (EC) method, Chip-packaging

\section{Introduction}

Recently, biosensors of nanoscale dimension have attracted wide attention for sensitive, multi-targeting, and labeling-free biomolecular detection. Various affinity-binding assisted analytical tools have been developed based on nanowires, nanoparticles, nanotubes. The widespread use of these tools, however, has been limited by random configuration of binding molecule and the formation of protein-protein cluster on a solid surface, resulting in a loss of masking of binding sites. Since the binding event is highly specific and and varies depending on the targeting strategy employeds, the biosensing platform needs to be flexible and constructed with many functional groups. To overcome this limit, we have developed nanowell arry structures for reducing nonspecific binding that highly affect to biosensor's sensitivity and selectivity. In this geometry, most of the area of the $\mathrm{Au}$ electrode is covered with a blocking layer, and only a nanosized gold surface is exposed to the open space above each nanowell array (NWA). The depth and width of the nanowells can be adjusted to allow only one or a few biomolecules to enter the well and become attached to the self-assembled modified gold surface. The number of wells required in a NWA to obtain the desired level of assay signal is easily estimated and an appropriated array can be fabricated.

Electrochemical (EC) detection was selected as analysis method that has high sensitivity compared with other classical analysis. We have reported that NWA can enhance EC detection of molecular binding events by controlling the binding sites of the captured molecules. Using NWA biosensor based direct current (DC) and Alternative current (AC) analysis, we have detected biological macromolecules such as DNA, protein as antigen or aptamers at low concentrations. ${ }^{1,2)}$ Moreover, miniaturization can be achieved by combining this simple EC method and nanosized electrode. The packaged device was tested for various type of biosensor. This system permits a much simpler packaging technique to be utilized and possesses advantages for many of the medical applications.

In this article, we will review a well-oriented NWA that is easily fabricated using current lithography technology and has numerous applications of biosensors. First, we will examine how to make this NWA structure by using various typed lithography. After this, we will also examine various applications of EC biosensors based on this NWA electrode. The area has been researched about our work through the use of on-line databases and browsing of journal content

Corresponding author

E-mail: he.lee@neu.edu

(C) 2014, The Korean Microelectronics and Packaging Society

This is an Open-Access article distributed under the terms of the Creative Commons Attribution Non-Commercial License(http://creativecommons.org/ licenses/by-nc/3.0) which permits unrestricted non-commercial use, distribution, and reproduction in any medium, provided the original work is properly cited. 
pages.

\section{Fabrication of Nanowell Array Electrode}

\subsection{Lithographic Molding}

Lithography molding is lithography for the fabrication of elastomeric stamps for microcontact printing $(\mu \mathrm{CP}){ }^{3)}$ This technique provides elastomeric stamps with sub-micrometer features and does not require routine access to clean rooms; it is thus an alternative to photolithography for the fabrication of stamps for $\mu \mathrm{CP}$. Lithographic molding combines several steps : i) microcontact printing on a thin gold film supported on a silicon (100) wafer to form a patterned SAM; ii) etching of the gold film, using the patterned self-assembled monolayers (SAMs) as resists iii) anisotropic etching of silicon which reduces the scale of certain features of the original pattern-in regions where removal of gold has exposed the underlying silicon wafer; iv) molding of a new elastomeric stamp using the etched silicon as a master, and v) $\mu \mathrm{CP}$ with this new elastomeric stamp. This demonstration is one of several that explore
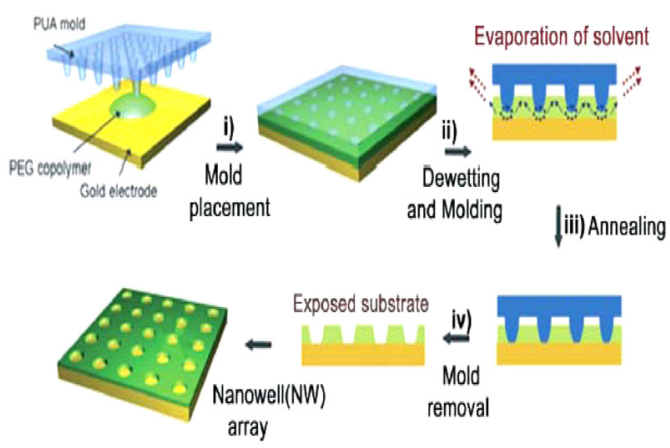

(a)
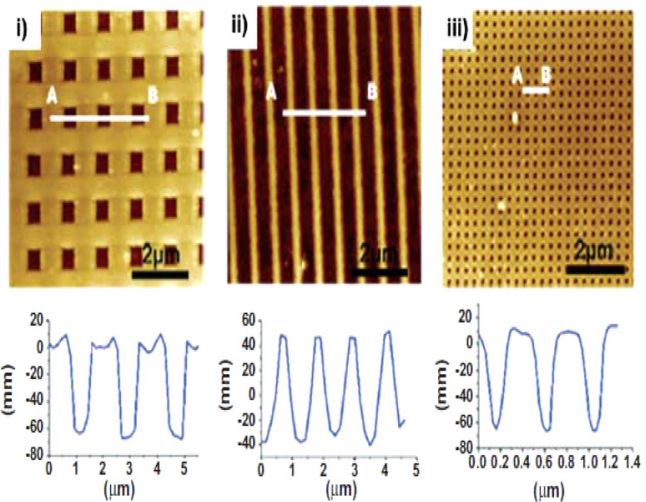

(b)

Fig. 1. (a) A schematic illustration for the fabrication of PEG nanowells with exposure of the substrate. (b) Planar and crosssectional AFM images of the fabricated PEG nanopatterns on gold substrate: i) Squares of $300 \mathrm{~nm}$ width and $70 \mathrm{~nm}$ height. ii) Lines of $200 \mathrm{~nm}$ width and $80 \mathrm{~nm}$ height. iii) Holes of $80 \mathrm{~nm}$ diameter and $70 \mathrm{~nm}$ height. Reproduced with permission from ref. number. alternatives to the photolithographic techniques normally used for the production of sub-micrometer scale features.

A schematic procedure for the fabrication of polyethylene glycol (PEG) NWA is shown in fig. 1. In this technique, a nanopattened poly metric mold is directly placed on a dropdispersed PEG solution before solvent evaporation. For the mold material, a UV-curable polyurethane acylate (PUA) was used, which was recently introduced for lithography of features smaller than $100 \mathrm{~nm}$. Using this method, various nanopatterns of a PEG random copolymer were fabricated using positive PUA molds with different feature size (Fig. 1b). AFM image demonstrated that these patterns were neatly constructed with the same lateral dimension with that of the original mold. The height varied depending on the geometry of the mold and the initial concentration/ amount of the polymer solution, ranging from 70 to $200 \mathrm{~nm}$. ${ }^{4}$

\subsection{Wafer-scale NWA patterning using KrF stepper}

In general, e-beam lithography (EBL), nanoimprint lithography (NIL) are capable of creating nanopatterns that can be used to fabricate NWA. But these methods have low throughput and are limited to small areas. We also used

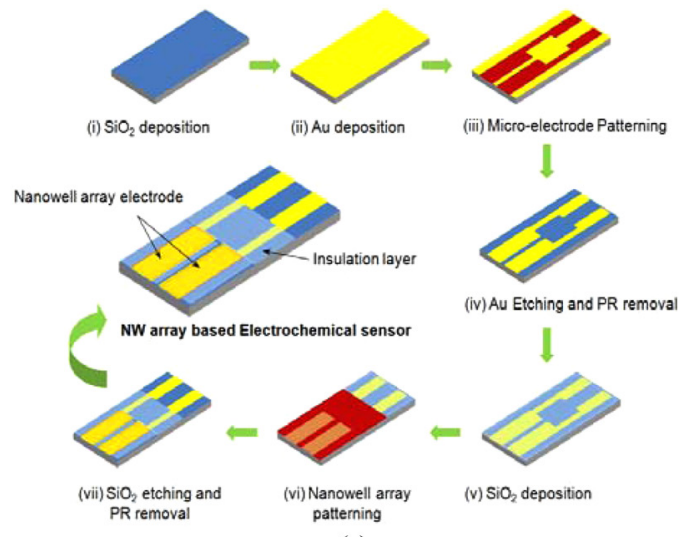

(a)

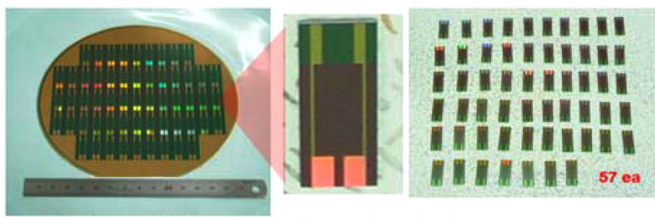

(I) As fabricated 6 inch wafer and a single sensor

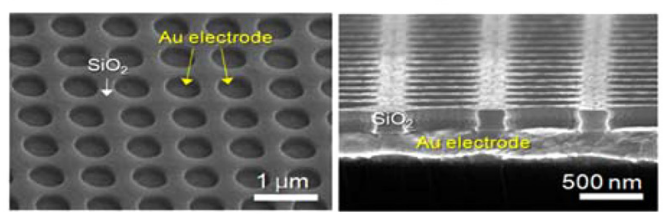

(I) FE-SEM images of Nanowell array electrode

(b)

Fig. 2. Fabrication of NWA based electrochemical sensor. (a) Simple and high-throughput nano process for industrial application. (b) Photograph of as-fabricated samples (top) and SEM images of NWA electrode (bottom). Reproduced with permission from ref. Num. 
krypton-fluoride $(\mathrm{KrF})$ stepper semiconductor process with a wavelength of $248 \mathrm{~nm}$ for the fabrication of NWA electrodes on 6 inch wafer. This wafer-scale fabrication method of nanopatterns has rapid, high-throughput and is highly reliable for the fabrication of NWA biosensor. As shown fig. 2 (a), a 6 inch wafer was pre cleaned using SPM (sulfuric peroxide mixture, $\mathrm{H} 2 \mathrm{SO} 4$ : $\mathrm{H} 2 \mathrm{O} 2=4: 1$ ) and diluted $\mathrm{HF}$ to remove the organic contaminants and native oxide layer on the substrate, respectively. A $300 \mathrm{~nm}$ thick $\mathrm{SiO} 2$ layer was deposited on the Si substrate by plasma-enhanced chemical vapor deposition (PECVD). Au layer was deposited by sputtering. After micro-patterning, the Ti/Au layers were etched in an ICP etcher. Finally, the exposed $\mathrm{SiO} 2$ areas were etched using an ICP etcher, and photoresist was removed. Also we designed nanowell array electrode with electronic pad for efficient packaging, Fig. 2 (b) shows the 57 uniform and well-fabricated NWA electrodes on 6-inch wafer fabricated by $\mathrm{KrF}$ stepper semiconductor process for industrial application. The size of a single NWA sensor was $21 \times 10 \mathrm{~mm} 2$. Each sensor consisted of two NWA with a size of $4 \times 2 \mathrm{~mm} 2$. Each NWA structure had a diameter of 500 $\mathrm{nm}$ with an interwell spacing of $200 \mathrm{~nm} .^{5)}$

\subsection{Chip-Level Packaging}

There exsist several challenges when developing the packaging process for the sensor chip. Some of these challenges are addressed in the sensor design stage such as sensor size and interconnections." ${ }^{6}$ The goal of the packaging process is to enable the sensor to its intended specifications but causing minimal effect to its environment and the sensor itself. Fig. 2 is also shown about our chipdesign for packaging. NWA (wide) and electronic pad (narrow) region are interconnected by $\mathrm{Au}$ pad. In order to reduce noise (that is most important factor for EC biosensor), we covered other region by PR that is insulating layer and reduce interconnection channel. Also we designed the chip to enable multi-targeting. Figure 3 shows the process for fabrication of a replica mold from Methacrylate octa-functionalized silsequioxane (SSQMA)-based formulation on a flexible and transparent poly-ethylene terephthalate (PET) film via UV-embossing. The duplicated pattern of parallel lines with a half-pitch of $50 \mathrm{~nm}$ showed alsmost the same half-pitch as the $\mathrm{Si}$ master mold. Rigid replicas duplicated onto a flexible backplane are called "rigiflex" replica molds. These results demonstrate that the SSQMAbased formulations are suitable for large-area replication with sub-50-nm features. ${ }^{7)}$

Rigiflex replica molds exhibit both local rigidity and global flexibility, which are advantageous in many applications. In

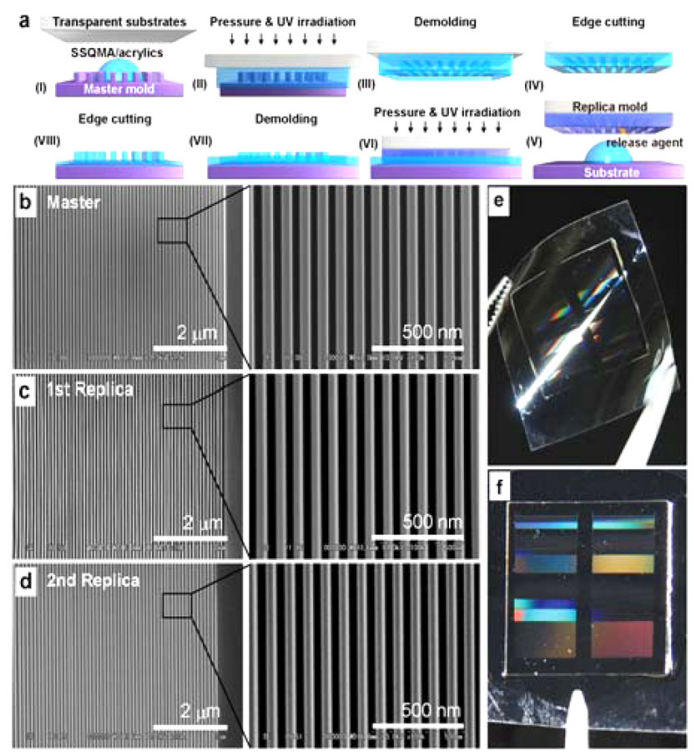

Fig. 3. (a) Schematic diagrams for the fabrication on PET film of a hybrid replica of a Si mold, using UV-NIL. FE-SEM images are given of (b) the Si master, (c) the first replica made with the SSQMA/EGDMA (50:50 wt \%) mixture on a flexible PET film, and (d) the second nanopatterned replica made with the SSQMA/MMA (50:50 wt \%) mixture on a glass substrate from the replica mold. Digital photographs are shown of (e) the rigiflex replica mold made with SSQMA/ EGDMA (50:50 wt \%) on a flexible PET film, and (f) the hard replica mold made with the SSQMA/MMA (50:50 wt $\%$ mixture on a glass substrate. Both areas of rigiflex and hard replica molds were $1 \times 1 \mathrm{~cm} 2$. Reproduced with permission from ref.

particular, even when the substrate is not flat, a flexible mold can provide large-area conformal contact with the substrate without requiring high pressures. This method demonstrated good reliability and performance. Also deposition, patterning and packaging are performed by simple process, this method has advantage of providing a continuous and high throughput. The cost of production can be dramatically reduced.

\section{NWA based Biosensor}

\subsection{Single Liposome based Sensor}

Nanoscale arrays of functional lipid vesicles (FLVs) are potentiaaly useful when employed in electrochemical studies and applications. Construction of such arrays on gold electrode with controlled dimension and density could allow for quantitative analysis on the level of single lipid vesicle with more reaction sites and low signal-tonoise ratio. To achieve a nanowell-based electrochemical biosensor with FLVs, three of the key directions are to address how to (I) capture FLVs at predefined locations without non-specific binding, (II) make the captured FLVs stable and fuctional, 
and (III) quantitatively interpret signals from affinity binding. Of these, non-specific binding has been a hurdle to most lavel-free detection methods since many proteins in a biological sample adhered to the surface non-specifically. Thus it would be great benefit to apply NWA electrode with controlled geometry and density that can capture individual liposomes without non-specific binding.

To evaluate potential applications of the site-selective deposition of single FLVs, we tested streptavidin binding to biotin on the FLVs surface. This coupling is widely studied for development of biosensor for its strong affinity. Fig. 4 (a) illustrates the streptavidin-biotin interactions using biotinylated FLVs containing thiol, ferrocence and PEG moieties on the surface of FLVs. Fig. 4 (b) shows SWV measurements for the change of current density on both electrodes (dash lines: before streptavidin-biotin binding, solid lines: after streptavidin-biotin binding). The SWV data demonstrated that the electrochemical responses (both magnitude and difference in peak current densities) were
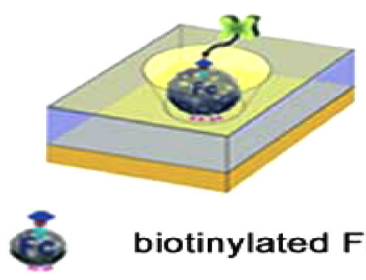

biotinylated FLVs

\section{Q streptavidin}

(a)
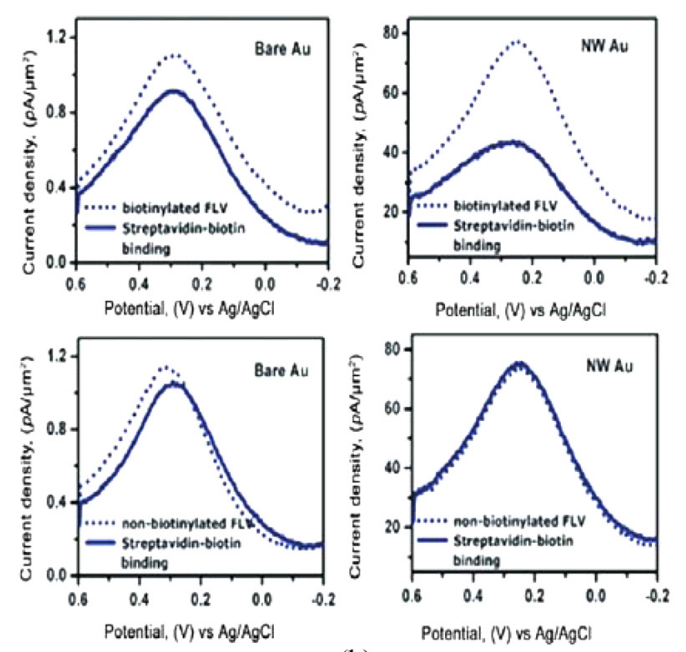

(b)

Fig. 4. (a) a schematic illustration of the binding event between streptavidin and captured biotinylated FLVs on NWA electrode. (b) SWV measurements of current density before and affter addition of streptavidin using a series of pulse input. The NWA electrode with non-biotinylated FLVs was used as the negative control. All the SWV data were obtained at a scan rate of $200 \mathrm{mV} / \mathrm{s}$. Reproduced with permission from ref.num. significantly enhanced for the NWA electrode: the difference in peak current densities (DIP) were $33.7 \mathrm{pA}$ $\mu \mathrm{m} 2$ and $0.187 \mathrm{pA} \mu \mathrm{m} 2$ for the NWA gold and the bare electrodes, respectively. Based on the active area exposed to the electrolyte, this difference corresponds to approximately 220 times increase in signal amplification for the NWA electrode, which could ultimately lead to a substantial increase in sensitivity.

\subsection{Electrochemical Immunosensor}

EIS (Electrochemical Impedance Spectroscopy) based immunosensors typically utilize antigen-antibody immunolayers that are very thin and have low electric permittivity. Molecular binding is detected by interruption of the faradaic current at the electrode, which generate an impedance signal. We developed an highly sensitive immunosensor for

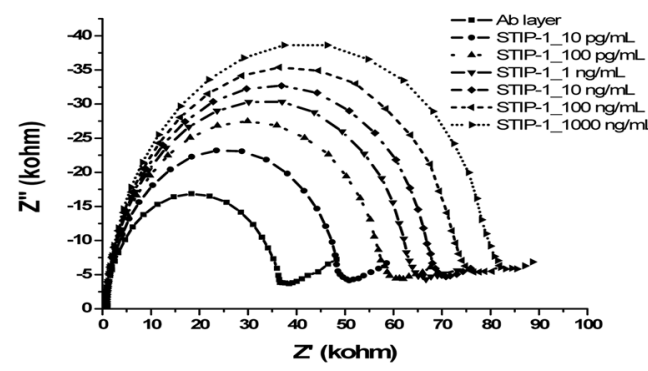

(a)

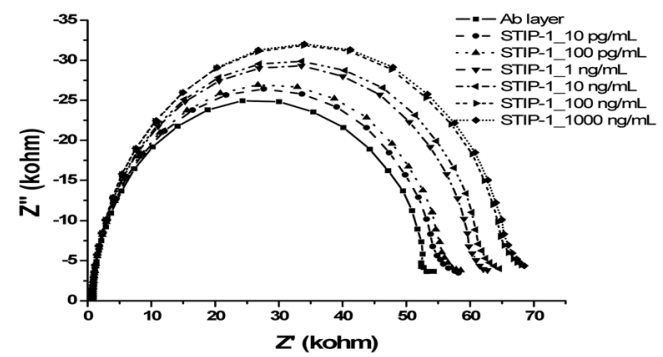

(b)

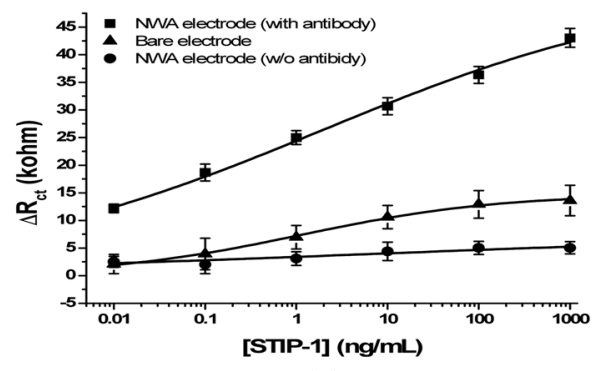

(c)

Fig. 5. EIS binding of STIP-1 to the immunolayer on the electrodes. (a) Nyquist plot for the NWA electrode. (b) Nyquist plot for the bare electrode. (c) Quantitative analysis of STIP-1 and anti STIP-1 antibody binding. Biotinylated anti-STIP-1 antibody layer was labeled with streptavidin on the NWA and bare electrodes. Error bars represent the standard deviations of three measurements. The applied potential was set to $50 \mathrm{mV}$ against an $\mathrm{Ag} / \mathrm{AgCl}$ reference electrode. The frequency range was $0.1 \mathrm{~Hz}$ to $1 \mathrm{MHz}$. Reproduced with permission from ref.Num. 
quantitative detection of specific antigen (stress-inducedphosphoprotein-1) [STIP-1] using NWA electrodes. Fig. 5 (a) and (b) show the Nyquist plot for NWA electrodes and bare electrodes without NWA using STIP-1 as a model analyte. In a nyquist representation, the real component of the complex impedance is shown on the x-coordinate, and the imaginary component on the y-coordinate. To compare the sensitivity of the NWA biosensor with that of other electrode-based biosensors, the same treatments were applied to a bare electrode without NWA. The LOD was estimated to be $10 \mathrm{pg} / \mathrm{mL}$ or less for the NWA electrodes, which suggests a 100-fold improvement in the LOD when using EIS with NWA electrode as shown fig. 5 (c). The sensitivity of the NWA impedimetric immunosensor was better for each analyte concentration tested when compared the sensitivity of the bare electrode sensor.

Based on these results, the electrochemical impedimetric immunosensors using NWA electrodes can be applied for label-free detection, with low levels of non-specific binding. Because NWA are optimal for the selective docking of single molecules, they reduce non-specific binding and enhance electrochemical responses. Therefore NWA have high sensitivity and selectivity as well as very low LOD.

\section{Conclusions}

In this article, we overviewed recent progress about NWA biosensors from its fabrication to its application. There are several potential advantages of NWA in biosensor applications. First, NWA can act as a digital assay by increasing the $\mathrm{S} / \mathrm{N}$ ratio. The nanowell geometry should minimize unwanted nonspecific binding and decrease the noise signal, since the resist layer can restrict many biomaterials from becoming attached to the EC active gold electrode surface. Second, the array can be used in numerous other integrated biosensor systems, such as in electronic signaling, fluorescence, and bioluminescence. Third, the fabrication of the NWA is highly compatible with recent advanced nanotechnologies and is quite economical. Also we designed the NWA by considering the packaging process. Improvement in the packaging process has resulted in a compact single-chip NWA electrode for reducing noise when measure the signal.

This type of array can be adapted relatively easily and used with a high throughput method. Finally, the NWA geometry should enhance the signal sensitivity so much that in the near future it might be feasible to detect a single biomolecular reaction. This nanometric system can also be applied to other integrated digital biosensors.

\section{Acknowledgements}

This research was supported by the Basic Science Research Program of the National Research Foundation of Korea (NRF), funded by the Ministry of Education, Science and Technology (2011-0014610).

\section{References}

1. J. M. Kim, H. S. Jung, J. W. Park, H. Oka, T. Yukimasa, H. Y. Lee and T. Kawai, "Spontaneous Immobilization of Liposomes on Electron Beam Technique", J. Am. Chem. Soc., 127(7), 2358 (2005).

2. H. S. Jung, J. M. Kim, J. W. Park, H. Y. Lee and T. Kawai. "Amperometric immunosensor for direct detection based upon functional lipid vesicles immobilized on nanowell array electrode", Langmuir : the ACS journal of surfaces and colloids., 21(13), 6025 (2005).

3. J. L. Wilbur, E. Kim and Y. Xia. "Whitesides GM. Lithographic molding: A convenient route to structures with submicrometer dimensions**", Advanced Materials., 7(7), 649 (1995).

4. P. Kim, B. K. Lee, H. Y. Lee, T. Kawai and K. Y. Suh. "Molded Nanowell Electrodes for Site-Selective Single Liposome Arrays", Advanced Materials., 20(1), 31 (2008).

5. J. Lee, S. Cho, H. Ryu, J. Park, S. Lim, B. Oh, C. Lee, W. Huang, A. Busnaina and H. Lee. "Wafer-scale nanowell array patterning based electrochemical impedimetric immunosensor", Journal of biotechnology., 168(4), 584 (2013).

6. D. C. Ng, T. Nakagawa, T. Mizuno, T. Tokuda, M. Nunoshita, H. Tamura, Y. Ishikawa, S. Shiosaka and J. Ohta. "Integrated in vivo neural imaging and interface CMOS devices: design, packaging, and implementation" Sensors Journal, IEEE., 8(1), 121 (2008).

7. B. K. Lee, N. G. Choa, H. Tanaka, N. Y. Hong, D. P. Kim, H. Y. Lee, T. Kawai. "Photocurable Polyhedral Oligomeric Silsequioxane-based Resists for Nanoimprint Lithography: Fabrication of High-aspect Ratio Structures and Replica Molds", Langmuir : the ACS journal of surfaces and colloids., 26(18), 14915 (2010). 

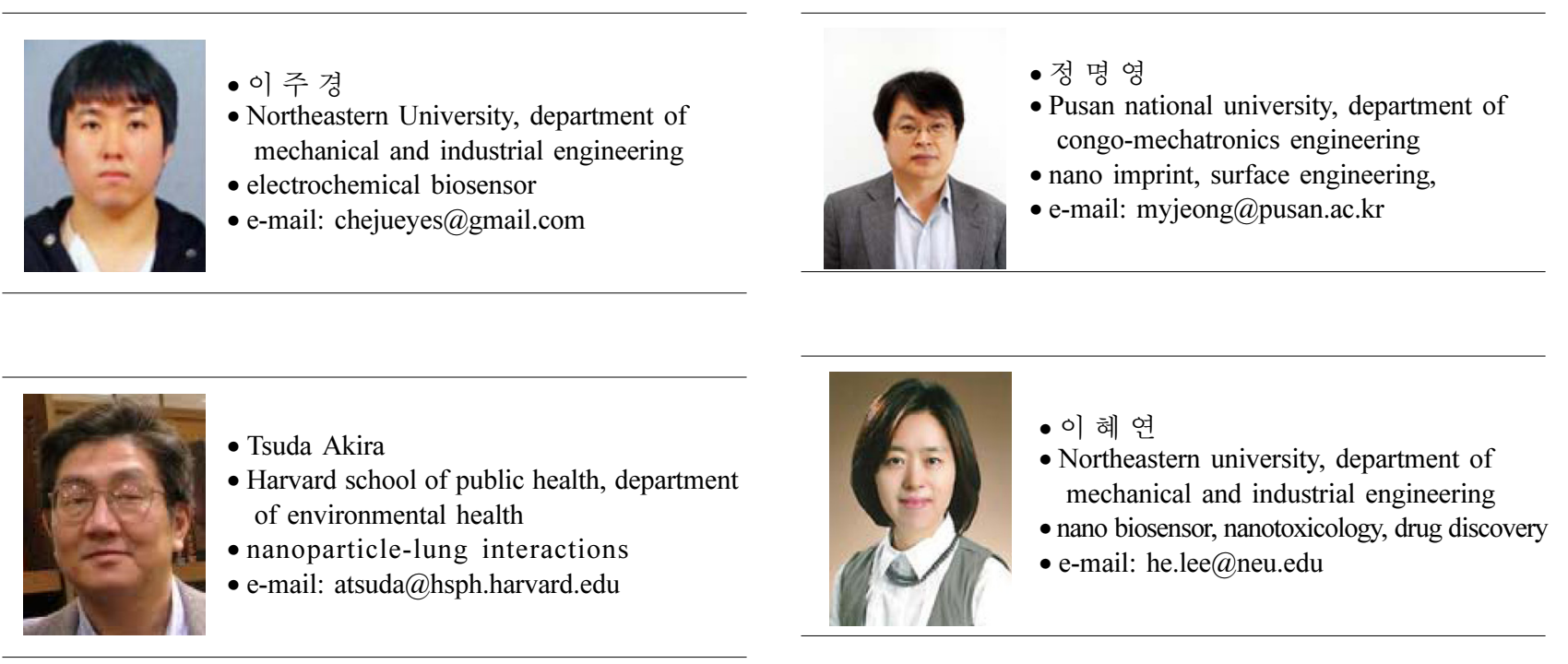